\title{
AVALIAÇÃO DO EFEITO DA TEMPERATURA E DA UMIDADE DO SUBSTRATO NA PRODUÇÃO DE (Hemi) CELULASES A PARTIR DA FERMENTAÇÃ̃O EM ESTADO SÓLIDO EM BIORREATOR DE COLUNAS INSTRUMENTADO
}

\author{
C.B. MELO $^{1}$, R.F. FONSECA ${ }^{1,2}$, V. BERTUCCI-NETO ${ }^{2}$, W.H. KWONG ${ }^{1}$, C.S. FARINAS ${ }^{1,2}$ \\ ${ }^{1}$ Universidade Federal de São Carlos, Departamento de Engenharia Química, São Carlos-SP \\ ${ }^{2}$ Embrapa Instrumentação, São Carlos-SP
}

E-mail para contato: rfrederico@gmail.com

\begin{abstract}
RESUMO - A avaliação da cinética da produção de enzimas (hemi) celulolíticas pode permitir o desenvolvimento de sistemas de controle mais eficientes para biorreatores de fermentação em estado sólido de larga escala. Por isso, o objetivo desse trabalho foi avaliar os efeitos da temperatura e da umidade inicial do substrato na cinética de produção de celulases e xilanases utilizando-se o farelo de trigo e o microrganismo A. niger (3T5B8). As atividades deCMCase e xilanase foram quantificadas em diferentes condições experimentais nos tempos de 24, 48 e 72 horas. Os resultados mostraram uma menor produção enzimática nas temperaturas mais alta $\left(46^{\circ} \mathrm{C}\right)$ e uma maior produção em $48 \mathrm{~h}$ nas temperaturas entre $\left(37^{\circ} \mathrm{C}\right.$ e $\left.28^{\circ} \mathrm{C}\right)$ e nas condições de maior umidade inicial do substrato $(51 \%$ a $66 \%$ ) em $72 \mathrm{~h}$. Para o estudo realizado, conclui-se que os sistemas de controle da temperatura do substrato em biorreatores de maior escala não necessitam operar o processo em uma estreita faixa operacional.
\end{abstract}

\section{INTRODUÇÃO}

Os primeiros registros dos processos fermentativos estão datados por volta de 6000 a.C. e descrevem a produção de cerveja, vinhos e pães na Babilônia no Egito. A cerca de 3000 a.C. iniciou-se a fabricação de shoyu na China e no Japão, de queijos e iogurtes nos Balcãs e na Ásia Central. Os processos fermentativos, ao longo da história até a $2^{\mathrm{a}}$ Guerra Mundial, eram utilizados em grande parte para a preservação de alimentos, transformação de suas propriedades nutricionais e produção de bebidas. A partir da descoberta da penicilina em 1928 por Alexander Fleming é quetais processos passaram a ser desenvolvidos, e mais amplamente por causa da demanda por antibióticos nos fronts das batalhas(Najafpour, 2007). Desde então, a Fermentação Submersa (FS) vem sendo empregada na produção de antibióticos e outros produtos. Essa escolha, em detrimento à Fermentação em Estado Sólido (FES), se deve principalmente às facilidades de controle das variáveis internas de um biorreator de FS quando comparado com os de FES. As variáveis mais importantes (e mais difíceis de serem controladas) da FES são a temperatura do meio durante a fermentação, o pH, a umidade do substrato e a aeração (oxigênio dissolvido) (Shaechter, 2004). 
Os processos de FES são caracterizados pela ausência de água livre, o que traz vantagens e desvantagens quando comparada à FS. Por ausência de água livre deve-se entender que a quantidade de água no meio não é suficiente para saturar o substrato, trataseda quantidade de apropriada para prover as necessidades microbianas. Uma das vantagens é a possibilidade de tratamento e reutilização de resíduos sólidos gerados pela agroindústria. Nesse caso, faz-se um pré-tratamento adequado aos resíduos, os quais são hidratados de modo a simular o habitat natural do microrganismo.

Por outro lado, a remoção do calor gerado pela respiração em biorreatores de grande porte é uma das dificuldades tecnológicas que ainda impedem o seu desenvolvimento em escala industrial (Holker e Lenz, 2005; Mitchell et al, 2006).Na literatura encontram-se alguns trabalhos que foram realizados com o objetivo de se controlar a temperatura no meio fermentativo, independentemente da produtividade de algum metabólito de interesse (Sargantanis et al., 1993), (Nagel et al., 2001). Outros modelaram a transferência de massa e de energia no interior de biorreatores (Lekanda e Perez-Correa, 2004), (Stuart e Mitchell, 2003), (Rahardjoet al, 2005), sendo que este último ainda modelou tanto o crescimento dos fungos quanto a transferência de água no interior das partículas sólidas. Observa-se em muitas referências grande variabilidade da temperatura no leito do biorreator ao longo do processo.

Segundo Mitchell et al(2006) o primeiro passo a ser dado no desenvolvimento de um processo de FES é a avaliação do mesmo em pequena escala, tal como frascos Erlenmeyer ou colunas de fermentação. Nesse procedimento, as principais variáveis são alteradas segundo um planejamento experimental para se averiguar a influência de cada uma na formação dos produtos desejados.

No Laboratório de Agroenergia da Embrapa Instrumentação - São Carlos - SP, foi desenvolvido de um biorreator do tipo colunas dotado de instrumentação e automação da umidade relativa do ar e da temperatura do ambiente. A principal característica desse instrumento é o controle preciso da umidade relativa do ar que alimenta o biorreator, passando de maneira forçada através do substrato, para uma faixa de temperaturas e fluxo de ar, respectivamente variando de $20^{\circ} \mathrm{C}$ a $46^{\circ} \mathrm{C}$ e de $0,5 \mathrm{vvm}$ a $2 \mathrm{vvm}$. Com esse sistema, algumas caracterizações dos processos de FES foram realizadas a fim de se avaliar a produção das enzimas celulolíticas em diferentes condições da aeração, umidade relativa, fluxo do ar e umidade inicial do substrato (UIS)(Farinas et al., 2011),(Vitcosque et al., 2012)e(Pirota et al., 2013). Os resultados apresentados nesses trabalhos apontam que para a maior parte dos fungos estudados a aeração ótima é cerca de $1 \mathrm{vvm}(20 \mathrm{~mL} / \mathrm{min})$ e umidade relativa do ar a $80 \%$. A produção das celulases ainda depende da temperatura do meio fermentativo, pois o crescimento microbiano varia com a temperatura do processo.

Nesse trabalho foi realizado um estudo da cinética de produção das enzimas CMCase e xilanase, avaliando-se a produção das mesmas de 24 em 24 horas durante as primeiras 72 horas do processo em diversas condições ambientais. Para tal, foi realizado um planejamento composto central (CCD) com 11 ensaios independentes, variando-se a temperatura do processo e a UIS, o queresultou em superfície de resposta da cinética das enzimas nas condições avaliadas.O objetivo primário dessa avaliação é a realização de uma modelagem matemática do processo a fim de se otimizá-lo quanto às condições iniciais e as condições ao longo do processo. No entanto, as superfícies de resposta apresentam resultados interessantes do ponto de vista de controle do processo. Por isso, o principal objetivo desse artigo foi avaliar quais as condições mais favoráveis na produção das enzimas estudadas e qual o 
melhor momento para se interromper o processo a fim de se obter a maior concentração das mesmas.

\section{MATERIAIS E MÉTODOS}

\subsection{Biorreator Instrumentado}

$\mathrm{O}$ biorreator de escala laboratorial utilizado consiste em 16 colunas $(2,5 \mathrm{~cm}$ de diâmetro, $20 \mathrm{~cm}$ de comprimento), climatizadas em banho térmico. $\mathrm{O}$ mesmo foi equipado com um sistema online de controle do fluxo e da umidade relativa do ar que alimenta as colunas. O algoritmo foi desenvolvido no Laboratório de Agroenergia e implementado pela interface LabView ${ }^{\circledR}$ 8.2., NationalInstruments, USA. Para esse trabalho, fixou-se em todos os cultivos, vazão de $20 \mathrm{~mL} / \mathrm{min}(1 \mathrm{vvm})$ e umidade relativa do ar de 80\%.As medidas da temperatura e da umidade relativa do ar foram feitas com uma sonda HMT330, Vaisala, Finland com precisão de $0,4^{\circ} \mathrm{C}$ e $1,5 \%$, respectivamente. $O$ controle da umidade relativa foi feito ajustando-seautomaticamenteaproporção entre ofluxo de ar saturado o fluxo total a fim de compensar o sinal da realimentação do sensor de umidade. Nessa etapa foi utilizado um controlador do tipo Proporcional Integral. As linhas de entrada de ar foram controladas pelo modelo GFM 17, Aalborg, USA no qual o sinal de referência varia de 0 a $5 \mathrm{~V}$ e o fluxo de ar varia de 0 a $686 \mathrm{~mL} / \mathrm{min}$, com erro de medida de $1,5 \%$. Um sensor foi utilizado para medir a pressão relativa da linha de alimentação de ar, modelo 26PC de 0 a 1 atm daHoneywell, USA. Todas as medidas realizadas foram gravadas em um computador através de um equipamento de aquisição de dados NI USB-6229, NationalInstruments, USA de 16 entradas referenciadas (ou 8 diferenciais) e 4 saídas analógicas para fins de comando externo, com período de amostragem de um minuto.Esse reatortambém possui um sistema de monitoramento do $\mathrm{CO}_{2}$ produzido durante o cultivo, realizado pelo sensor GMM221, Vaisala, com sensibilidade de 0 a $5 \%$.

\subsection{Fermentação em Estado Sólido (FES) para Produção de Enzimas}

O fungo utilizado foi oAspergillus niger (3T5B8), mutante da coleção da EmbrapaTecnologia de Alimentos. Este mutante foi obtido por técnicas convencionais de mutação induzida usando agentes químicos e físicos por (Couri e Defarias, 1995). A cultura foi mantida em tubos em meio PDA a temperatura ambiente, sob fina camada de óleo mineral. Conduziu-se a FES nas colunas do biorreator instrumentado citado anteriormente, contendo aproximadamente $10 \mathrm{~g}$ de farelo de trigo. Após a inoculação dos esporos ( $10^{7}$ esporos/g) foi adicionado uma quantidade sempre fixa de solução nutriente de Sulfato de Amônio 0,91\% em $\mathrm{HCl}$ 0,1 M com diferentes volumes de água para obtenção de UISconforme o planejamento experimental proposto em base úmida.

\subsection{Extração do Meio Enzimático}

Após o período de cultivo, foram adicionados ao material fermentado tampão acetato de sódio $0,1 \mathrm{M}, \mathrm{pH}$ 4,8 na relação de $1: 10(\mathrm{~m} / \mathrm{v})$, sendo homogeneizado e posteriormente agitado por $30 \mathrm{~min}$, a $200 \mathrm{rpm}$. O material foi, posteriormente, filtrado e centrifugado a $10000 \mathrm{rpm}$ durante $20 \mathrm{~min}, \mathrm{a} 4^{\circ} \mathrm{C}$, e o sobrenadante utilizado como solução enzimática bruta. 


\subsection{Atividades Enzimáticas e Métodos Analíticos}

A atividade da xilanase foi medida em mistura de reação contendo $1 \mathrm{~mL}$ de extrato enzimáticoe $2 \mathrm{~mL}$ de solução de xilana $1 \%$ em tampão acetato 0,2 $\mathrm{M}$ e pH 5,0.Após incubação a $50{ }^{\circ} \mathrm{C}$ por 30 minutos, os açúcares redutores foram quantificados pelo método DNS (Miller, 1959).Procedimento semelhante foi utilizado para a atividade de CMCase, onde o substrato utilizado foi $1 \mathrm{~mL}$ de solução $4 \%$ de CMC em tampão citrato de sódio $50 \mathrm{mM}$ e pH 4,8 com o tempo de incubação de 10 minutos.

\subsection{Planejamento Experimental}

O planejamentodesse trabalhoconsiste no uso da metodologia da superfície de resposta a partir do delineamento composto central (CCD) com 11 ensaios independentes conforme a Tabela 1. Até o presente momento, o ensaio 11 não foi realizado e no ensaio 10 estão faltando os valores das amostras de 72 horas.

Tabela 1 - Ensaios do planejamento.

\begin{tabular}{|c|c|c|c|c|c|c|c|}
\hline \multirow{2}{*}{\multicolumn{3}{|c|}{ Ensaio }} & \multicolumn{5}{|c|}{ Umidade Substrato } \\
\hline & & & $\begin{array}{c}-1,41 \\
36 \% \\
\end{array}$ & $\begin{array}{c}-1 \\
40 \%\end{array}$ & $\begin{array}{c}0 \\
51 \% \\
\end{array}$ & $\begin{array}{c}+1 \\
62 \% \\
\end{array}$ & $\begin{array}{r}+1,41 \\
66 \% \\
\end{array}$ \\
\hline \multirow{5}{*}{$\begin{array}{l}\frac{\sigma}{J} \\
\frac{1}{\pi} \\
\frac{\pi}{2} \\
\frac{0}{E} \\
\frac{\varepsilon}{\varrho} \\
1\end{array}$} & +141 & $46^{\circ} \mathrm{C}$ & & & 6 & & \\
\hline & +1 & $43^{\circ} \mathrm{C}$ & & 8 & & 11 & \\
\hline & 0 & $37^{\circ} \mathrm{C}$ & 5 & & 1,2 e 3 & & 7 \\
\hline & -1 & $31^{\circ} \mathrm{C}$ & & 9 & & 10 & \\
\hline & $-1,41$ & $28^{\circ} \mathrm{C}$ & & & 4 & & \\
\hline
\end{tabular}

Os cultivos foram realizados em triplicatas independentes para cada período de tempo (24h, 48h e 72h),com o objetivo de se estudar a cinética de produção enzimática do fungo A. niger no substrato farelo de trigo.As análises enzimáticas, realizadas conforme referências citadas anteriormente foram analisadas em triplicatas independentes por coluna.

\subsection{Avaliação dos Resultados}

A avaliação dos resultados foi feita em duas etapas. A primeira foi a análise do produto das médias da produção das enzimas em cada ensaio. Esse procedimento foi adotado de modo a se penalizar uma baixa produção de uma das enzimas, poisse considera que melhores resultados de uma futura hidrólise são conseguidos quando existe uma concentração maisalta deambas as enzimas.Na segunda etapa, o resultado da primeira foianalisado através da metodologia da superfície de respostaspara otimização de processos, através do software Statistica $\bigcirc$, ignorando-se adequadamente os efeitos das interações entre variáveis que não foram significativas.

\section{RESULTADOS E DISCUSSÃO}

A Figura 1, (a), (b) e (c), mostra a superfície de resposta da cinética do produto 
daprodução das enzimasnas amostras de 24, 48 e 72 horas.Essas superfícies foram obtidas através da interpolação cúbica por partes dos valores obtidos dos produtos entre as enzimas. A Figura 2 (a), (b) e (c), mostra a superfície de reposta da análise dos 3 fatores na produção das enzimas: o tempo, a temperatura do banho e a UIS. A Figura 3 mostra o gráfico de Pareto dos efeitos dos fatores na produção enzimática, que são os valores das constantes da Equação (1).

Figura 1 - Gráfico do produto das atividades enzimáticas $\left(\frac{U I}{g}\right)^{2} \mathrm{x}$ Temperatura $\left({ }^{\circ} \mathrm{C}\right) \mathrm{x}$ Umidade inicial do substrato em: $24 \mathrm{~h}$ (a), $48 \mathrm{~h} \mathrm{(b)} \mathrm{e} 72 \mathrm{~h}$ (c). A barra lateral das figuras indica a cor referente ao produto das atividades enzimáticas de cada nível da superfície de resposta.

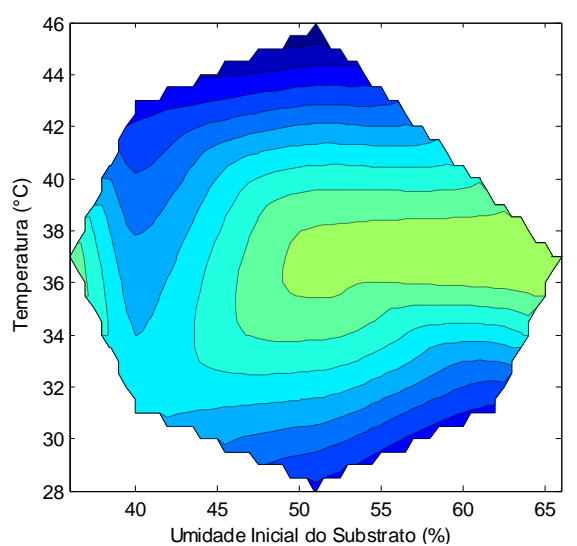

(a)
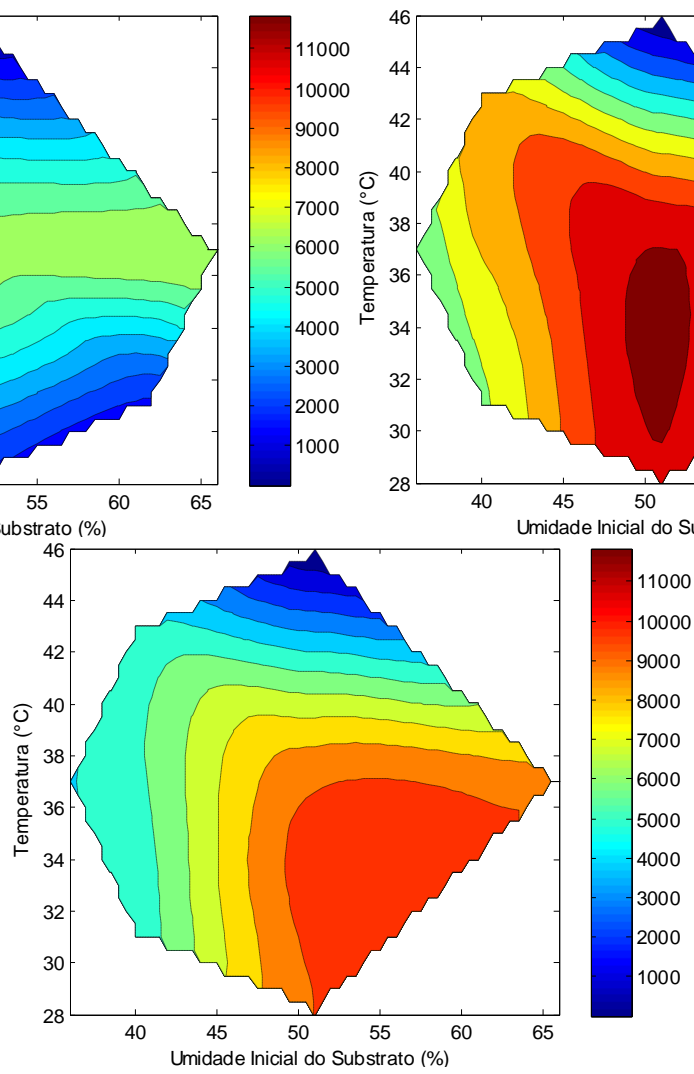

(c)

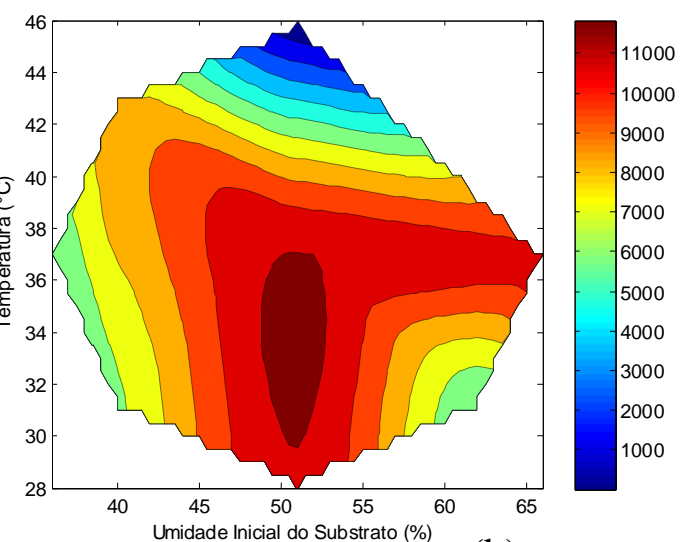

(b)

A Equação (1) define a superfície de resposta da segunda etapa da avaliação dos resultados. Nas equações, $x, y$ e $z$ representam, respectivamente, os fatores tempo, temperatura e umidade inicial do substrato.

$$
P=\alpha_{0} x^{2}+\alpha_{1} x+\alpha_{2} y^{2}+\alpha_{3} y+\alpha_{4} z^{2}+\alpha_{5} z+\alpha_{6} x y+\alpha_{7} x z
$$

Onde $\alpha_{i}$ são constantes. 
Figura 2 - Superfície de resposta do efeito daTemperatura $\left({ }^{\circ} \mathrm{C}\right)$, da Umidade inicial do substrato e do Tempo (h) no produto das atividades enzimáticas $\left(\frac{U I}{g}\right)^{2}$. Em (a) Temperatura $\left({ }^{\circ} \mathrm{C}\right) \times$ Tempo (h), em (b) Umidade inicial do substratoxTempo (h) e em (c) Temperatura $\left({ }^{\circ} \mathrm{C}\right)$ xUmidade inicial do substrato.

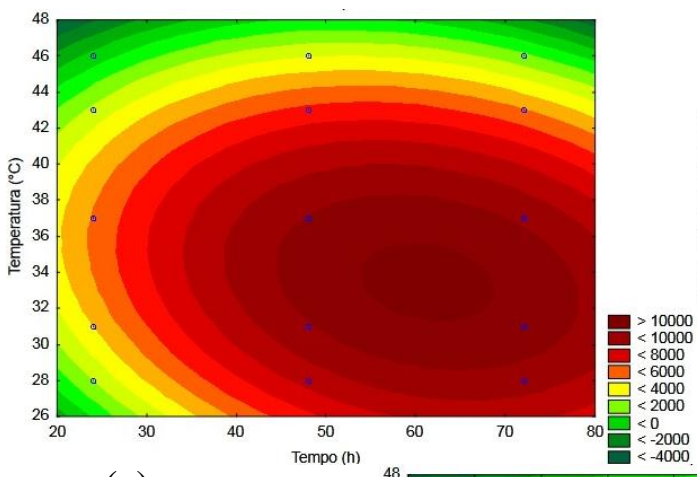

(a)

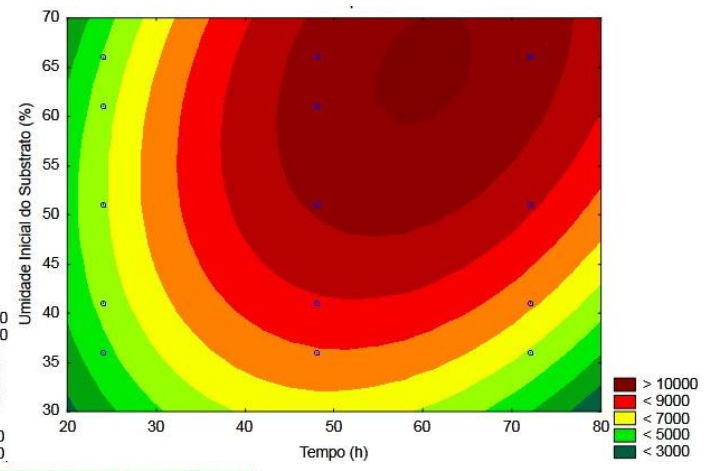

(b)

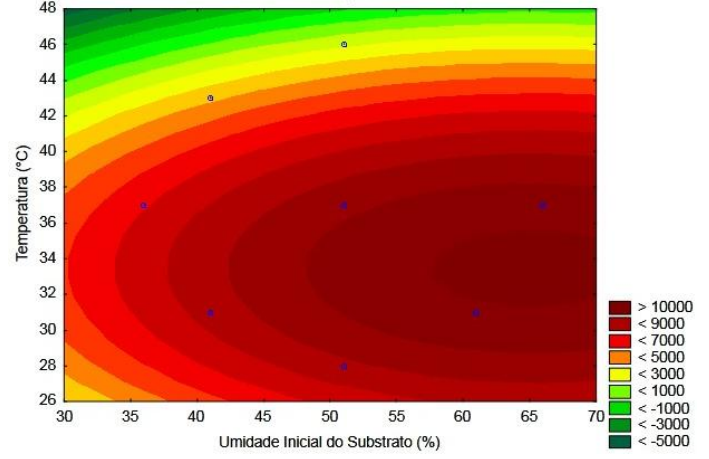

(c)

Figura 3 - Gráfico de Pareto para análise dos efeitos dos fatores na produção das enzimas.

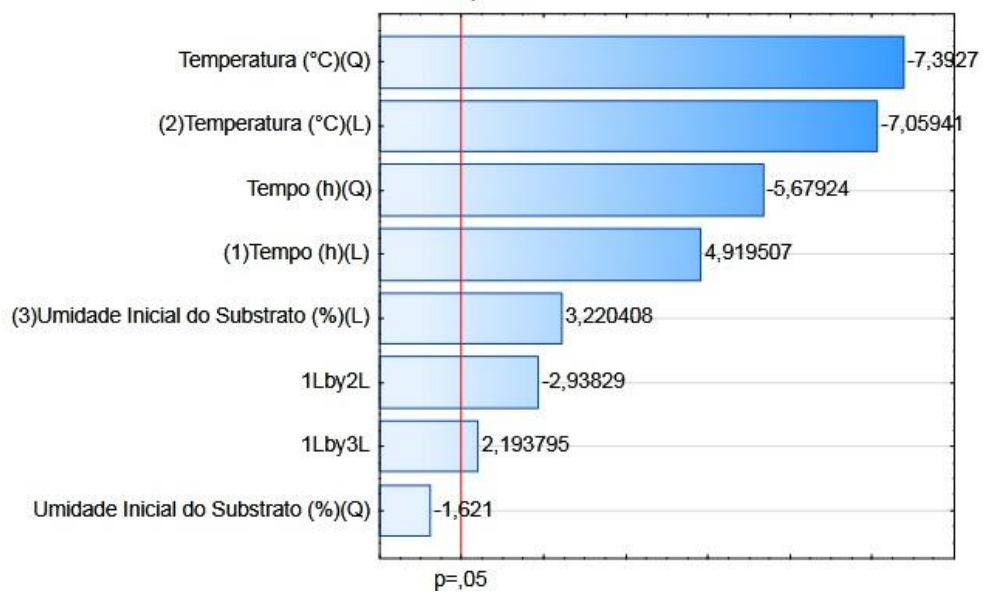

Observa-se claramente que na Figura 1 a maior produção das enzimas (produto entre ambas) ocorre nas amostras de 48 horas, e ainda, que nas proximidades da condição central de UIS a produção é bastante alta para todas as temperaturas inferiores a $39^{\circ} \mathrm{C}$. Esse resultado é corroborado com as informações do gráfico da Figura 3 que mostra que o aumento da temperatura tem efeitos negativos na produção das enzimas.

A Figura (2),(a) e (b), aponta que a maior produção das enzimas deve ocorrer no 
tempo de 61 horas, com temperatura de $33^{\circ} \mathrm{C}$ e UIS de $65 \%$. A Figura também aponta que altos resultados são o obtidos com 48 horas com temperaturas entre $32^{\circ} \mathrm{C}$ e $37^{\circ} \mathrm{C}$ e com umidades iniciais do substrato acima de 51\%, o que pode ser visto na Figura 2 (c). Além disso, visto que a temperatura tem maior impacto sobre o processo do que a UIS, torna-se mais fácil validar esses resultados na região central da umidade do que no extremo superior. Essa consideração é reforçada pelo resultado do ensaio 10, que demonstra uma menor produção das enzimas quando comparado com as umidades centrais.

\section{CONCLUSÕES}

Apesar de inicialmente o objetivo do planejamento experimental realizado ter sido a modelagem matemática do processo, muitas inferências qualitativas puderam ser feitas cujas conclusões guiarão alguns dos futuros trabalhos de controle referentes a biorreatores de maior porte. Conforme pode ser observado, existe uma região de temperatura e umidade do substrato, na qual a produção das enzimas é bastante elevada. Considerando que uma das maiores dificuldades apresentadas na FES é a heterogeneidade do meio, abre-se espaço para um controle menos restritivo do ambiente do processo. Assim sendo, o objetivo do sistema de controle não seria mais especificar uma temperatura fixa, mas uma faixa de temperaturas apropriadas para uma boa produção dos metabólitos. Com isso, para biorreatores de leito agitado e tambor rotativo, o controlador deve compensar a distribuição da temperatura e da umidade do meio, levando-se em conta os efeitos da agitação e da aeração no processo. Além disso, é sabido que em biorreatores de FES a metodologia mais empregada na remoção do calor é a do resfriamento evaporativo, técnica que retira calor juntamente com a umidade do meio. Também, conforme apontado nesse artigo, existe uma tendência a uma boa produção dos metabólitos com UIS maiores. Assim sendo, entende-se que a fermentação em um biorreator de maior porte pode ser inicializada com uma UIS maior e que ao longo do processo, mesmo com as perdas por evaporação, a mesma ainda pode ser mantida na faixa aponta pela Figura 1, sem necessidade de reposição de água do meio.

\section{REFERÊNCIAS}

COURI, S.; DEFARIAS, A. X. Genetic manipulation of Aspergillus niger for increased synthesis of pectinolytic enzymes. Revista De Microbiologia, v. 26, n. 4, p. 314-317, Oct-Dec 1995. ISSN 0001-3714.

FARINAS, C. S. et al. Modeling the effects of solid state fermentation operating conditions on endoglucanase production using an instrumented bioreactor. Industrial Crops and Products, v. 34, n. 1, p. 1186-1192, Jul 2011. ISSN 0926-6690.

HOLKER, U.; LENZ, J. Solid-state fermentation - are there any biotechnological advantages? Current Opinion in Microbiology, v. 8, n. 3, p. 301-306, Jun 2005. ISSN 1369-5274.

LEKANDA, J. S.; PEREZ-CORREA, J. R. Energy and water balances using kinetic modeling in a pilot-scale SSF bioreactor. Process Biochemistry, v. 39, n. 11, p. 1793-1802, Jul 2004. ISSN 1359-5113.

MILLER, G. L. Use of Dinitrosalicylic Acid Reagent for Determination of Reducing Sugar. Analytical Chemistry, v. 31, n. 3, p. 426-428, 1959. ISSN 0003-2700. 
MITCHELL, D. A.; KRIEGER, N.; BEROVIC, M. Solid-State Fermentation Bioreactors. Springer-Verlag, 2006. 480p.

NAGEL, F. et al. Temperature control in a continuously mixed bioreactor for solid-state fermentation. Biotechnology and Bioengineering, v. 72, n. 2, p. 219-230, Jan 2001. ISSN 0006-3592.

NAJAFPOUR, G. D. Biochemical Engineergin and Biotechnology. Elsevier, 2007. 439p.

PIROTA, R. D. P. B. et al. Enhancing xylanases production by a new Amazon Forest strain of Aspergillus oryzae using solid-state fermentation under controlled operation conditions. Industrial Crops and Products, v. 45, p. 6, 2013.

SAKURAI, Y.; LEE, T. H.; SHIOTA, H. CONVENIENT METHOD FOR GLUCOSAMINE ESTIMATION IN KOJI. Agricultural and Biological Chemistry, v. 41, n. 4, p. 619-624, 1977 1977. ISSN 0002-1369.

SARGANTANIS, J. et al. EFFECT OF OPERATING-CONDITIONS ON SOLID SUBSTRATE FERMENTATION. Biotechnology and Bioengineering, v. 42, n. 2, p. 149158, Jun 1993. ISSN 0006-3592.

SHAECHTER, M. The Desk Encyclopedia of Microbiology. Elsevier Academic Press, 2004. 1169.

STUART, D. M.; MITCHELL, D. A. Mathematical model of heat transfer during solid-state fermentation in well-mixed rotating drum bioreactors. Journal of Chemical Technology and Biotechnology, v. 78, n. 11, p. 1180-1192, Nov 2003. ISSN 0268-2575.

VITCOSQUE, G. L. et al. Production of Biomass-DegradingMultienzyme Complexes under Solid-State Fermentation of Soybean Meal Using a Bioreactor. Enzyme Research, v. 2012, p. $9 \mathrm{p}, 2012$. 